\title{
Equine Mesenchymal Stromal Cells Retain a Pericyte-Like Phenotype
}

\author{
Cristina L. Esteves,, Tara A. Sheldrake,, Lucy Dawson,, Timothy Menghini,, Burgunde Elisabeth Rink, \\ Karin Amilon, ${ }^{1}$ Nusrat Khan, ${ }^{2}$ Bruno Péault, ${ }^{2,3}$ and Francesc Xavier Donadeu ${ }^{1}$
}

Mesenchymal stem/stromal cells (MSCs) have been used in human and equine regenerative medicine, and interest in exploiting their potential has increased dramatically over the years. Despite significant effort to characterize equine MSCs, the actual origin of these cells and how much of their native phenotype is maintained in culture have not been determined. In this study, we investigated the relationship between MSCs, derived from adipose tissue (AT) and bone marrow (BM), and pericytes in the horse. Both pericyte (CD146, NG2, and $\alpha$ SMA) and MSC (CD29, CD90, and CD73) markers were detected in equine AT and colocalized around blood vessels. Importantly, as assessed by flow cytometry, both pericyte (CD146, NG2, and $\alpha \mathrm{SMA}$ ) and MSC (CD29, CD44, CD90, and CD105) markers were present in a majority ( $\geq 90 \%)$ of cells in cultures of AT-MSCs and BM-MSCs; however, levels of pericyte markers were variable within each of those populations. Moreover, the expression of pericyte markers was maintained for at least eight passages in both AT-MSCs and BM-MSCs. Hematopoietic (CD45) and endothelial (CD144) markers were also detected at low levels in MSCs by quantitative polymerase chain reaction (qPCR). Finally, in coculture experiments, AT-MSCs closely associated with networks produced by endothelial cells, resembling the natural perivascular location of pericytes in vivo. Our results indicate that equine MSCs originate from perivascular cells and moreover maintain a pericyte-like phenotype in culture. Therefore, we suggest that, in addition to classical MSC markers, pericyte markers such as CD146 could be used when assessing and characterizing equine MSCs.

Keywords: mesenchymal stem cells, equine, horse, adipose tissue, bone marrow, pericyte

\section{Introduction}

$\mathrm{H}$ ORSES AND HUMANS are the two species in which interest in the therapeutic use of mesenchymal stem/stromal cells (MSCs) is highest. In the horse, the past decade saw a dramatic increase in the use of MSC preparations [1,2], in particular for repair of musculoskeletal injuries [3-6], whereby conventional treatments have limited efficacy. The main clinical application of equine MSCs has been tendon and ligament repair [7-10], but other conditions such as joint disease and laminitis are also being considered [11]. Importantly, because musculoskeletal pathophysiology in horses resembles that in humans, the U.S. Food and Drug Administration has approved the use of horses as a large animal preclinical model, which broadens the relevance of the findings obtained in equine.

Equine MSCs used clinically are mainly derived from bone marrow (BM) and adipose tissue (AT) $[2,12,13]$. BM samples are processed using a density gradient to isolate the mononucleated cell fraction, whereas AT extracts are obtained by collagenase digestion [14]. Both BM and AT extracts contain heterogeneous cell populations that can be cultured and characterized for their MSC properties. Contrary to human MSCs, there are no standard guidelines to define equine MSCs and, therefore, the minimum criteria established in 2006 by the International Society for Cellular Therapy (ISCT) are commonly applied [15]. Specifically, ISCT guidelines state that MSCs must grow adherent to plastic and have the ability to differentiate into adipocytes, osteocytes, and chondrocytes. Guidelines for adipose-derived MSC surface markers have been more recently updated [16] to include CD44 in addition to CD105, CD73, and CD90, while CD45 and CD31 must be absent. In relation to equine MSCs, although trilineage differentiation is usually demonstrated, a variable immunophenotype is often reported for cells of different tissue origins, and additional makers such as CD29 are being considered to characterize MSCs [1,17-21].

\footnotetext{
${ }^{1}$ The Roslin Institute, University of Edinburgh, Edinburgh, United Kingdom.

${ }_{3}^{2}$ Centre for Regenerative Medicine, University of Edinburgh, Edinburgh, United Kingdom.

${ }^{3}$ Orthopaedic Hospital Research Centre, University of California, Los Angeles, California.
}

(C) Cristina L. Esteves et al. 2017; Published by Mary Ann Liebert, Inc. This is an Open Access article distributed under the terms of the Creative Commons Attribution License, which permits unrestricted use, distribution, and reproduction in any medium, provided the original work is properly cited. 
Despite significant work to characterize their properties, the precise in vivo origin of equine MSCs and whether they maintain their original phenotype in culture have not been determined. Pericytes are perivascular cells, also known as Rouget or mural cells, and are embedded in the basement membrane of small blood vessels, capillaries, and microvessels [22-24]. In humans, isolated pericytes expressing CD146, NG2, PDGFR $\beta$, and $\alpha$ SMA have been described as a native source of MSCs [25], but it is still not clear how the pericyte phenotype is maintained in cultured MSCs obtained from heterogeneous cell extracts of AT and BM.

To further understand the nature of equine MSCs, in this study we investigated the relationship between equine MSCs and pericytes both in native tissues and in culture, including a comparison between AT-MSCs and BM-MSCs.

\section{Materials and Methods \\ Samples}

Samples were obtained immediately postmortem from eight adult horses. All animal procedures were carried out according to the U.K. Home Office Animals (Scientific Procedures) Act 1986 with approval by the Ethical Review Committee, University of Edinburgh. Tissues (AT, BM, and large blood vessels) were kept on ice until cell extraction or were frozen in cold isopentane mounted in optimum cutting temperature compound (OCT; VWR Chemicals, Leicestershire, United Kingdom) for immunohistochemistry (IHC). Subcutaneous AT was obtained by dissection from the abdominal region close to the linea alba.

\section{Immunohistochemistry}

Samples were processed in a Leica CM1900 cryostat, allowed to air dry, and processed right away or kept frozen at $-80^{\circ} \mathrm{C}$. For staining, tissues were fixed in ice cold acetone-methanol (50:50) and antibodies were prepared in diluent (003118; Invitrogen-Thermo Fisher Scientific, Paisley, United Kingdom) and, after staining, slides were mounted in fluoroshield with DAPI (Sigma-Aldrich, St Louis, MO). Primary antibodies used in the study are listed in Table 1

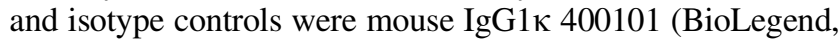
San Diego, CA), mouse IgG1 MCA928F (AbD SerotecBioRad, Kidlington, United Kingdom), mouse IgG1 MAB002
(R\&D Systems, Minneapolis, MN), mouse IgG1к 557273 (BD, Oxford, United Kingdom), and rabbit PRABP01 (AbD Serotec-BioRad). Secondary antibodies were AF488 conjugated (A11008 and A11029) and AF568 conjugated (A110037 and A10042). Isotypes and secondary antibodies alone were used as controls. Micrographs were produced using a Zeiss LSM710 confocal or Leica DMLB fluorescent microscope.

\section{Cell extraction and culture}

AT samples were minced and digested for $45 \mathrm{~min}$ with collagenase II (1 mg/mL; Gibco-Thermo Fisher Scientific)/ BSA $(3.5 \%)$ at $37^{\circ} \mathrm{C}$ under agitation $(100 \mathrm{rpm})$. Collagenase activity was stopped by addition of Dulbecco's modified Eagle's medium with $20 \%$ fetal bovine serum (FBS) (Thermo Fisher Scientific) and the lipid layer was removed after separation by gravity. BM-MSCs were obtained by aspiration of sternum marrow followed by centrifugation on a density gradient, and the mononucleated cell layer was harvested. Endothelial cells were harvested by tying up both ends of a small piece of blood vessel that had been washed with phosphate-buffered saline (PBS) and filled with collagenase II $(1 \mathrm{mg} / \mathrm{mL})$. After a $45-\mathrm{min}$ digestion, endothelial cells were collected and cultured.

To determine colony-forming unit-fibroblasts (CFU-F), cells were seeded at a density of 1,5 , and 25 cells $/ \mathrm{cm}^{2}$ and allowed to grow for 11 days in tissue culture dishes of $55 \mathrm{~cm}^{2}$ growth surface area. Colonies were observed by staining for phosphatase alkaline activity using a commercial kit (86R1KT; Sigma-Aldrich). In brief, cells were washed twice with PBS, then fixed with paraformaldehyde-citrate acetate buffer for $30 \mathrm{~s}$, and washed again with PBS, to which the alkaline dye mixture was added.

\section{RNA extraction and gene expression analyses}

Cultured cells were harvested in Trizol (Thermo Fisher Scientific) and RNA was reverse transcribed to cDNA using SuperScript III (18080-044; Invitrogen-Thermo Fisher Scientific). Quantitative polymerase chain reaction (qPCR) was performed using SensiFAST SYBR Lo-ROX kit (Bioline, London, United Kingdom) and equine primers designed by us, specifically to recognize PDGFR $\beta$ (5'-GTGCCTACAAAG GCTCCCAT-3' and 5'-CACAGTGGGATCTGGCACAA-3'),

Table 1. List of Antibodies Used in This Study

\begin{tabular}{|c|c|c|c|c|c|}
\hline Antibody & Reactivity & Company & Cat. number & Conjugate & Clone \\
\hline CD29 & Human, cow & BioLegend & $303002 / 303015$ & Purified/AF488 & $\mathrm{TS} 2 / 16$ \\
\hline CD44 & Horse & AbD Serotec & MCA1082GA & Purified & CVS18 \\
\hline CD73 & Rat & $\mathrm{BD}$ & 551123 & Purified & $5 \mathrm{~F} / \mathrm{B} 9$ \\
\hline CD90 & Rat & $\mathrm{BD}$ & $554895 / 561973$ & Purified/FITC & OX7 \\
\hline CD105 & Human, horse, monkey & AbD Serotec & MCA1557T & Purified & SN6 \\
\hline CD144 & Human, mouse, rat, cow & AbD Serotec & AHP628Z & Purified & Polyclonal \\
\hline CD146 & Human, pig & Biorad & MCA2141F & FITC/AF647 & OJ79c \\
\hline NG2 & Human & $\mathrm{R} \& \mathrm{D}$ & $\begin{array}{l}\text { MAB2585/ } \\
\text { FAB2585A }\end{array}$ & Purified/APC & LHM-2 \\
\hline$\alpha \mathrm{SMA}$ & $\begin{array}{l}\text { Mouse, rat, chicken, } \\
\text { guinea pig, cow, } \\
\text { dog, human }\end{array}$ & Abcam & ab5694 & Purified & Polyclonal \\
\hline
\end{tabular}


CD144 (5'-TCTGCAGGACATCAATGACAAC- $3^{\prime}$ and $5^{\prime}$ CTTCAG GCACGGCAAATACG-3'), NG2 (5'-CGCATCA TTGGGCCCTACTT-3' and 5'-GCTGTTCCACCTCTCTCC AG-3'), and 18S (5'-GCTGGCACCAGACTTG-3' and 5'GGGGAATCAGGGTTCG-3'), or obtained from the literature [20]. Reactions proceeded in a MX3005P qPCR system (Stratagene, CA). Results were analyzed with Stratagene MxPro software and normalized to $18 \mathrm{~S}$ levels quantified in the same samples.

\section{Flow cytometry}

Flow cytometry was performed in samples of AT-MSCs and BM-MSCs stained at $4^{\circ} \mathrm{C}$ with primary antibody for $1 \mathrm{~h}$ and, when necessary, with secondary antibody for $30 \mathrm{~min}$. Primary antibodies are listed in Table 1. Secondary antibodies were AF488 conjugated (A11029; Invitrogen), AF405 conjugated (Ab175654; AbCam, Cambridge, United Kingdom), or APCCy7 conjugated (sc-3847; Santa Cruz Biotechnologies, Paso Robles, CA).

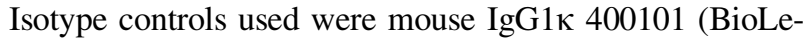
gend), mouse IgG1 MCA928A647 (AbD Serotec-BioRad), mouse IgG1 MAB002, and IgG1 IC002A (R\&D Systems), or rabbit PRABP01 (AbD Serotec-BioRad). Sytox blue (Thermo Fisher Scientific) was used as live cell stain. Iso- types and secondary antibodies alone were used as controls. Samples were run on a BD LSRFortessa or BD FACSAria Fusion (BD Biosciences, San Jose, CA) and data were analyzed on FlowJo_V10 (LLC, Ashland).

\section{Cell differentiation}

AT-MSCs and BM-MSCs were differentiated into adipocytes, osteocytes, and chondrocytes. Adipogenesis was induced by DMEM/F-12 (Life technologies) containing 7\% rabbit serum (Gibco-Thermo Fisher Scientific), 3\% FBS (Invitrogen), 1\% penicillin/streptomycin (Gibco-Thermo Fisher Scientific), $1 \mu \mathrm{M}$ dexamethasone (Sigma Aldrich), $0.5 \mathrm{mM}$ IBMX (Sigma Aldrich), $10 \mu \mathrm{g} / \mathrm{mL}$ insulin (Sigma Aldrich), and $100 \mu \mathrm{M}$ indomethacin (Sigma Aldrich). Adipocytes were stained with Oil Red O (Sigma-Aldrich) as described before [26]. Chondrogenesis and osteogenesis were induced using StemPro differentiation kits following manufacturer's instructions for 18 days (A10071-01 and A10072-01, respectively; Life Technologies). Chondrocyte pellets were embedded in Histogel (HG-4000; Invitrogen) and paraffin, and cut at $8 \mu \mathrm{m}$ thickness. Slides were dewaxed and rehydrated and Alcian blue solution (1\%) was added to the slide and incubated overnight. Then, the stain was removed and the slides were washed. Neutral red $(1 \%)$
FIG. 1. IHC of equine AT sections showing staining for the pericyte markers, CD146 (A), NG2 (B), $\alpha$ SMA (C), and the MSC markers, CD29 (D) and CD90 (E). CD144 was used as endothelial marker (red; A, B, D, E). Colocalization of pericyte (NG2 and CD146) and MSC markers (CD146 and $\mathrm{CD} 73 ; \mathbf{F}, \mathbf{G}$, respectively) in yellow (left panel) from the overlap of green (middle panel) and red (right panel) individual antibody fluorescence. DAPI was used to stain nuclei. Scale bar, $10 \mu \mathrm{m}$, is indicated by white bars. IHC, immunohistochemistry; MSC, mesenchymal stem/stromal cell. Color images available online at www .liebertpub.com/scd
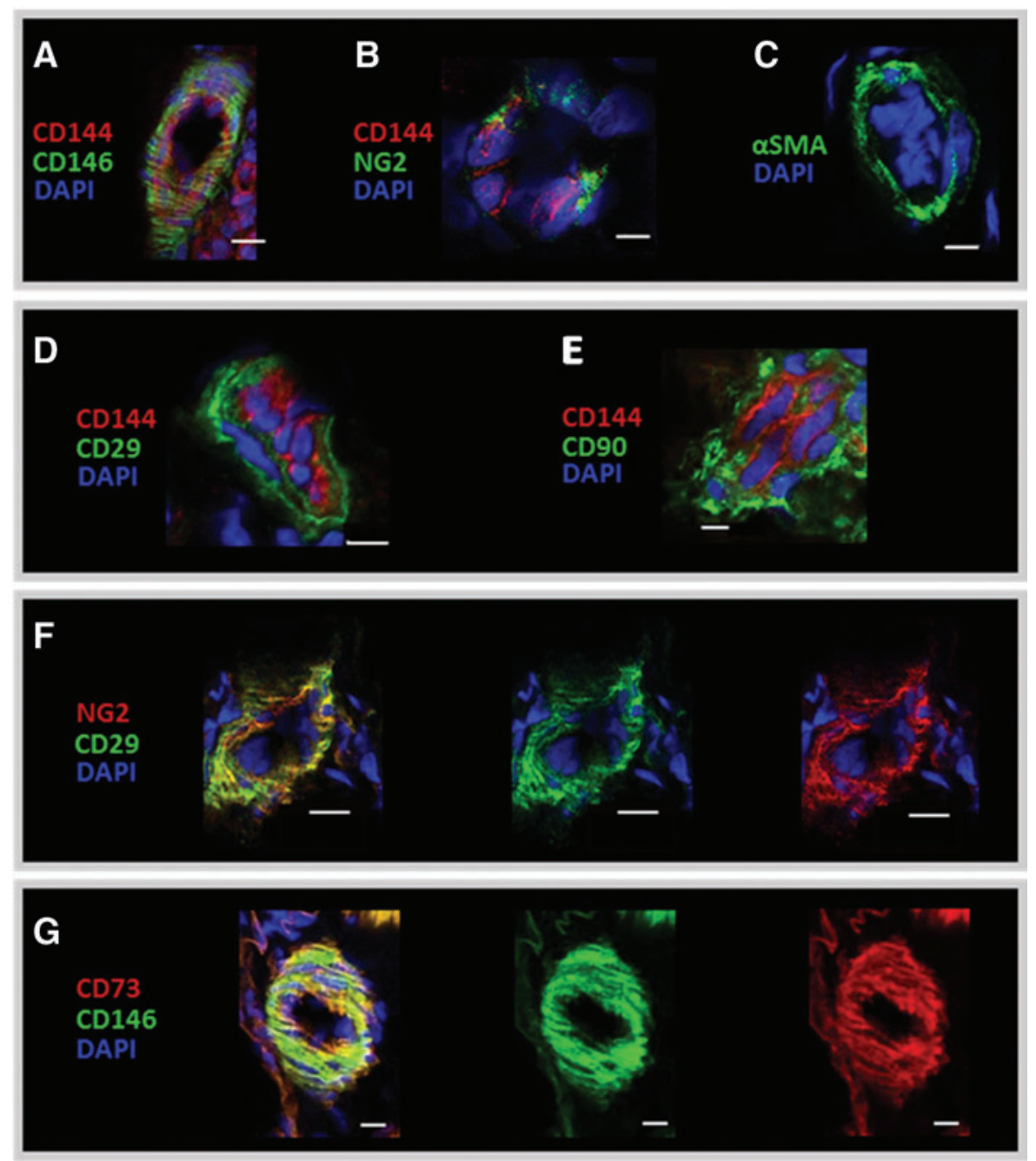
solution was added and incubated for $1 \mathrm{~min}$ and rinsed in water. Absolute ethanol and xylene were used to dehydrate the slides that were mounted with pertex. Osteogenic cultures were washed with PBS and fixed with PFA (4\%) for $15 \mathrm{~min}$ and stored in PBS at $4^{\circ} \mathrm{C}$. After washing with water, Alizarin Red $\mathrm{S}$ (A5533; Sigma-Aldrich) prepared in water with $\mathrm{pH}$ adjusted to 4.2 was added to cover the cellular monolayer. The plate was incubated at room temperature in the dark for $10 \mathrm{~min}$ and washed with water. Negative controls were produced with nondifferentiated cells and micrographs were taken in an Axiovert 25 Inverted Microscope (Zeiss, Oberkochen, Germany).

\section{Angiogenesis}

The fluorescent dyes PKH26 $(20 \mu \mathrm{M})$ and PKH67 $(20 \mu \mathrm{M})$ (both from Sigma-Aldrich) were used to label AT-MSC (red) and equine endothelial cells (green). Once labeled, cells were resuspended in EGM-2 medium and seeded on ibiTreat $\mu$ Slides (IB-81506; Thistle Scientific) coated with matrigel. Pictures were taken using a Zeiss Live Cell Observer/ Deconvolution system.

\section{Statistical analysis}

Results are shown as mean \pm standard error of the mean, and were analyzed by Students' $t$-test or two-way analysis of variance followed by Tukey test using GraphPad Prim 6.0 software (GraphPad Software, La Jolla, CA). Significance was set at $P<0.05$.

\section{Results}

\section{Localization of pericytes in equine $A T$}

The relative location of pericyte (CD146, NG2, and $\alpha$ SMA) and MSC (CD29, CD73, and CD90) markers was examined by immunohistochemistry in equine adipose or testis (Fig. 1 and Supplementary Fig. S1; Supplementary Data are available online at www.liebertpub.com/scd). CD146 and NG2 were detected around small blood vessels as evidenced by the presence of adjacent endothelial cells stained with CD144 (Fig. 1A, B) in agreement with previous results from human samples $[25,27]$. Other cell types in the tissue (Supplementary Fig. S1A, B) were negative for these pericyte markers, as well as the isotypes and secondary antibody controls (Supplementary Fig. S1D-F), showing cross-reactivity by CD146 and NG2 antibodies. $\alpha$ SMA was also perivascular (Fig. 1C), whereas none of the five different PDGFR $\beta$ antibodies tested gave a specific signal (data not shown). For the classical MSCs, antibodies tested before in equine tissues [19], were also located around small blood vessels, as shown by the results of CD29 and CD90 (Fig. 1D, E). In addition, dual staining with antibodies against NG2 and CD29, or CD146 and CD73 (Fig. 1F, G and Supplementary Fig. S1B) confirmed the colocalization of pericytes and MSC markers in equine AT and in testis, consistent with the notion that pericytes may give rise to MSCs in culture.

\section{Characterization of AT-MSCs and BM-MSCs}

Extracts from equine AT and BM were obtained by collagenase digestion and density gradient, respectively, and the resulting cells grew in uncoated tissue culture vessels, showing a characteristic spindled-like morphology (Fig. 2A). To test the ability of AT-MSCs and BM-MSCs to form CFU-Fs, cells were
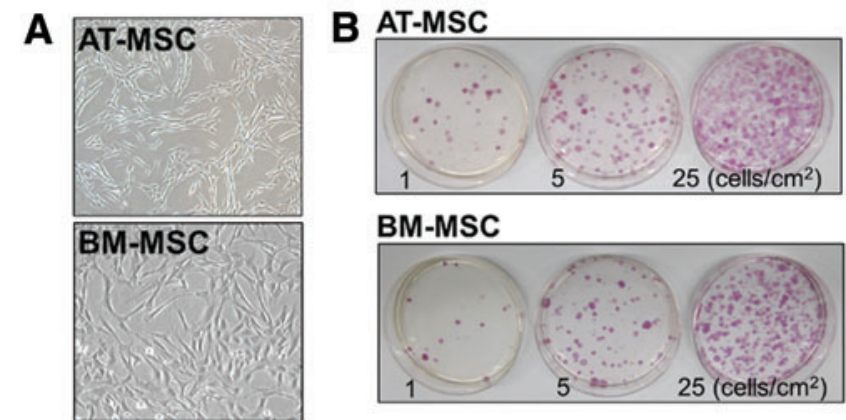

BM-MSC
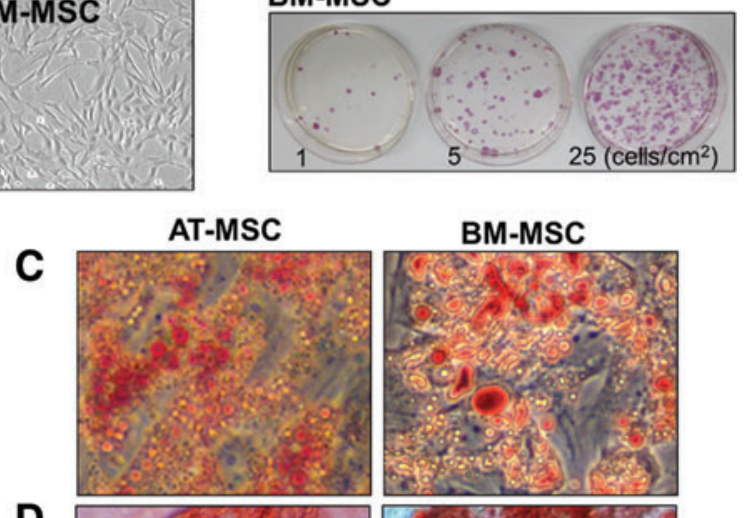

D
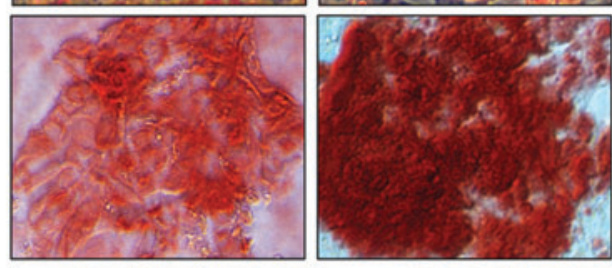

E
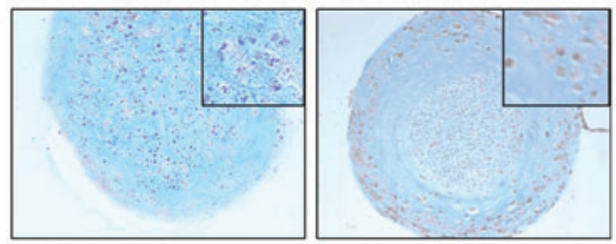

FIG. 2. Photomicrograph of cultured AT-MSCs and BMMSCs (A) that generated AP-positive colonies when grown at low density $\left(1,5\right.$, and 25 cells $\left./ \mathrm{cm}^{2}\right)$ for 11 days $(\mathbf{B})$ and differentiated into adipocytes (C), osteocytes (D), and chondrocytes (E) observed by Oil Red O, Alizarin Red S, and Alcian blue counterstained with Neutral red, respectively. Inset shows detail of the chondrocyte micromasses. AT, adipose tissue; BM, bone marrow. Color images available online at www.liebertpub.com/scd

seeded at 1,5 , and 25 cells $/ \mathrm{cm}^{2}$. After 11 days, both AT-MSCs and BM-MSCs formed colonies $(49.5 \pm 13.9$ and $43.5 \pm 10.5$ CFU-F, respectively, for cells seeded at a density of 5 cells $/ \mathrm{cm}^{2}$ ), which were positive for alkaline phosphatase (Fig. 2B), as reported before for human MSCs [28] and pericytes [25,29].

Both AT-MSCs and BM-MSCs were multipotent. Adipogenic capacity was evidenced by Oil Red $\mathrm{O}$ staining, showing accumulation of lipids in the cytoplasm (Fig. 2C), osteogenesis was evidenced by the dark red staining of calcium deposits produced by Alizarin Red S (Fig. 2D) and chondrogenesis was shown by Alcian blue staining of cartilage matrix in cell micromasses (Fig. 2E).

The expression of typical MSC markers was confirmed by flow cytometry (Fig. 3A) by using antibodies that have previously been validated or used in other equine studies $[19,20]$. The results showed that $\geq 99 \%$ of the cells were positive for CD29, CD44, CD90, and CD105 in both ATMSCs and BM-MSCs. qPCR analyses confirmed comparable expression of CD44, CD73, CD90, and CD105 by the 
A AT-MSC
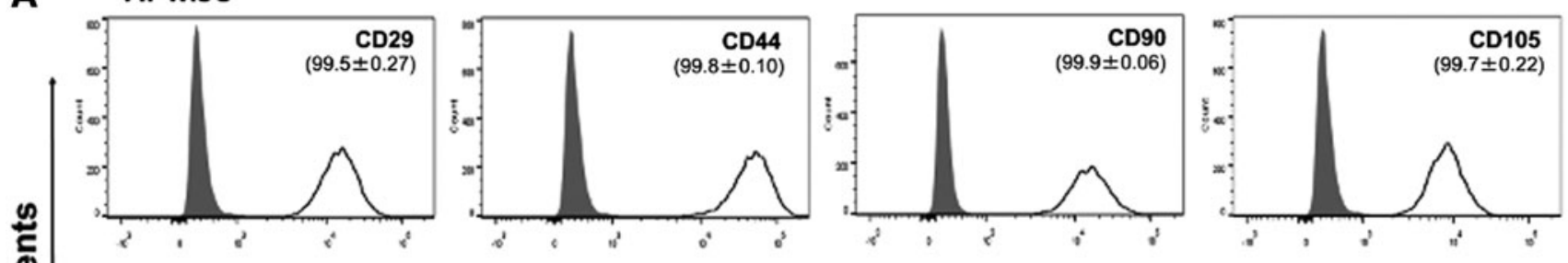

BM-MSC
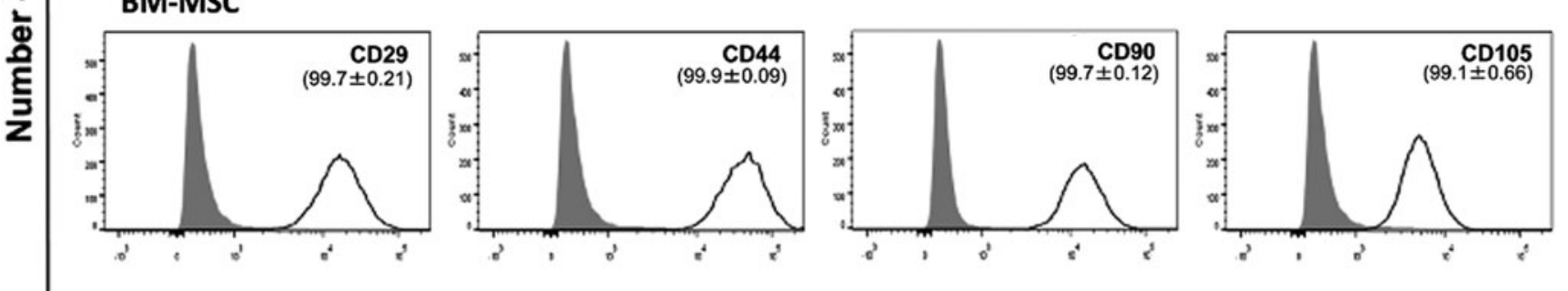

Fluorescence Intensity

B

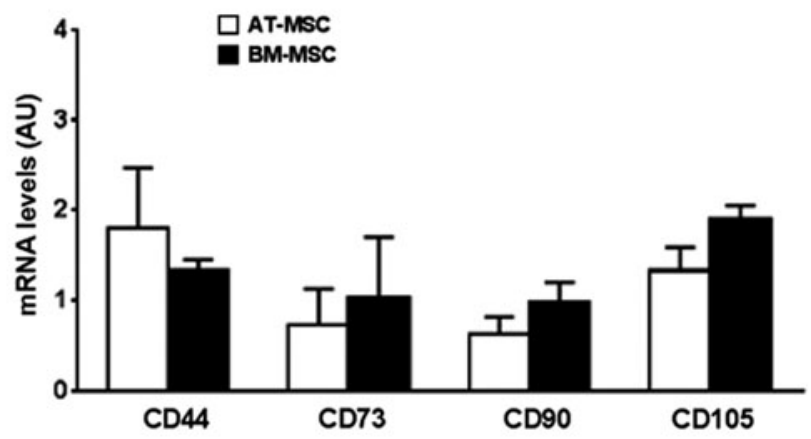

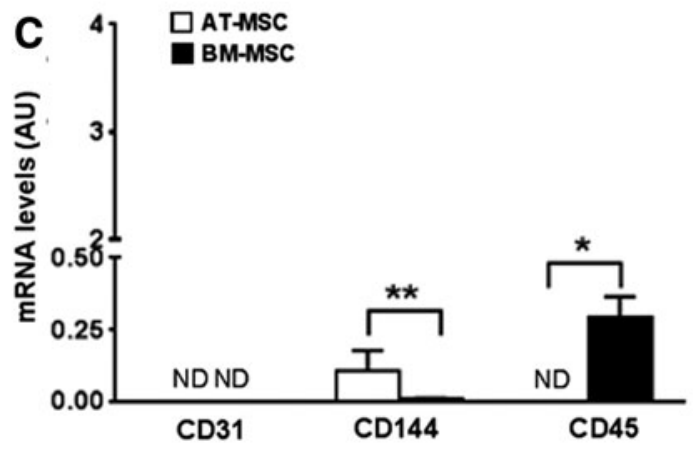

FIG. 3. Flow cytometry histograms of AT-MSCs and BM-MSCs (A; upper and lower panels, respectively) showing positive staining of most cells for the MSC markers, CD29, CD44, CD90, and CD105 (dark line; $\geq 99 \%$ cells for all antibodies by detection of AF488 conjugate or FITC conjugate). Signal from isotype controls is shown by the gray peak and unstained curves were omitted for simplicity. (B) Results of quantitative polymerase chain reaction (qPCR) analysis of the MSC markers, CD44, CD73, CD90, and CD105, and (C) the endothelial markers, CD31 and CD144, and the hematopoietic marker, CD45, in AT-MSCs and BM-MSCs. All results are shown as mean \pm SEM; $n=3$ animals. $* P<0.05$ and $* * P<0.005$. AU, arbitrary units; ND, not detected; SEM, standard error of the mean.

two cell types (Fig. 3B). Moreover, the hematopoietic maker, CD45, was not detected in AT-MSCs but was present at low levels in all BM-MSC cultures. Low levels of expression of the endothelial cell marker, CD144, were observed in both AT-MSCs and BM-MSCs, whereas CD31 was not detected. Overall, these results showed that our cells fulfilled the criteria for MSCs and confirmed that AT-MSC and $\mathrm{BM}-\mathrm{MSC}$ preparations are heterogeneous in nature.

\section{AT-MSCs and BM-MSCs express pericyte markers in culture}

Following from our finding that MSC and pericyte markers colocalize in equine tissues (Fig. 1), we wanted to determine whether the expression of pericyte markers would be maintained by MSCs in culture by using complementary data from flow cytometry and qPCR, and to do this we validated further the pericyte antibodies (Supplementary Fig. S2). Flow cytometry showed the pericyte markers, CD146 and NG2, to be highly expressed ( $\geq 94.6 \%)$ in ATMSCs and BM-MSCs, whereas $\alpha$ SMA was present at slightly lower levels ( $\geq 89.9 \%$ ). Histograms showed a broad distribution in the fluorescence intensity of CD146- and NG2-positive cells, suggesting highly variable expression among individual cells (Fig. 4A). To confirm these findings and at the same time determine whether pericyte markers were maintained by MSCs during extended culture, we quantified the expression of CD146, NG2, and PDGFR $\beta$ by qPCR in AT-MSCs and BM-MSCs at passages 2 and 8 (Fig. 4B). All pericyte markers were expressed at similar levels in AT-MSCs and BM-MSCs, and although CD146 and PDGFR $\beta$ levels remained unchanged across passages in both MSC types, NG2 expression decreased $(P<0.05)$ at passage 8 in BM-MSCs.

\section{MSCs adopt a periendothelial location in vitro}

To assess whether MSCs maintain functional characteristics of perivascular cells, we cocultured AT-MSCs and endothelial cells on matrigel. In endothelia-only cultures, a cell network was incipient after seeding and became increasingly organized with dense strands (Fig. 5). AT-MSCs 

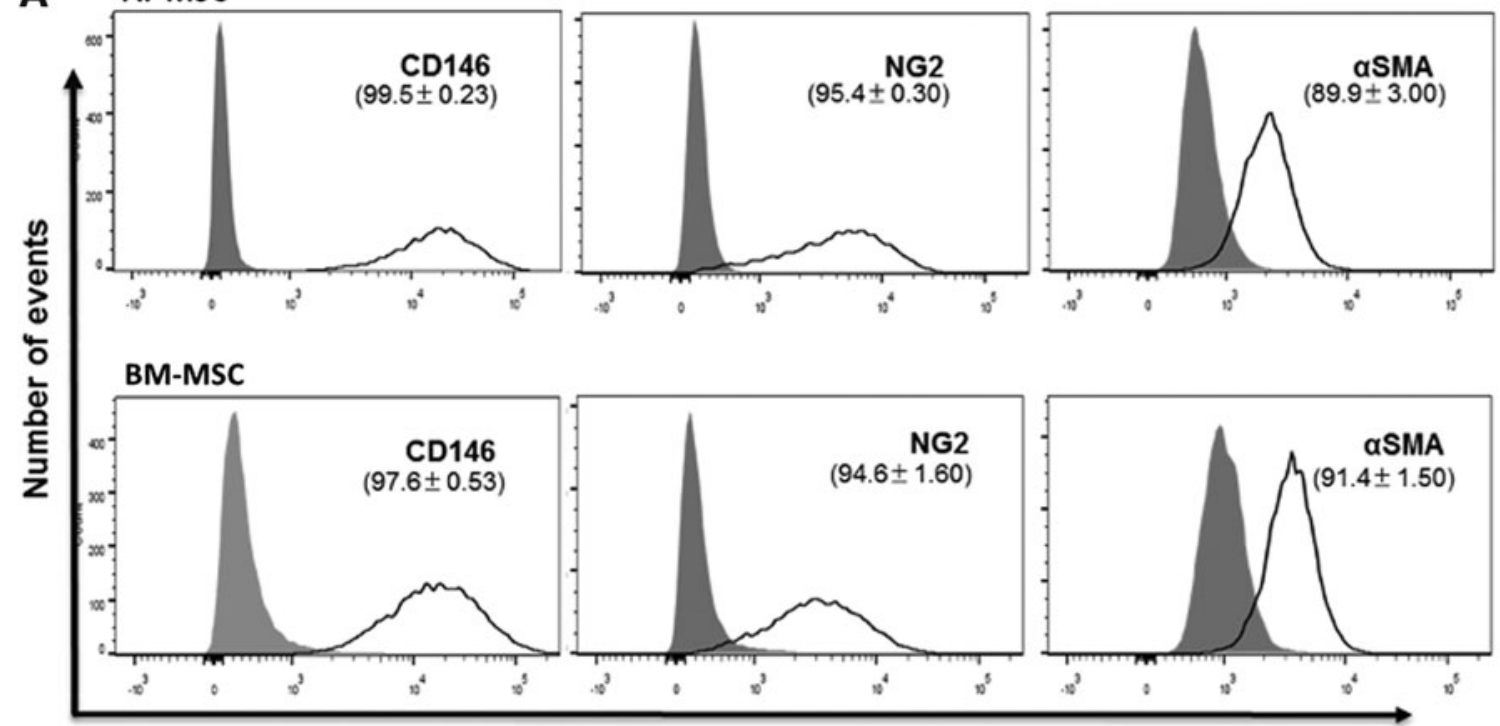

Fluorescence Intensity
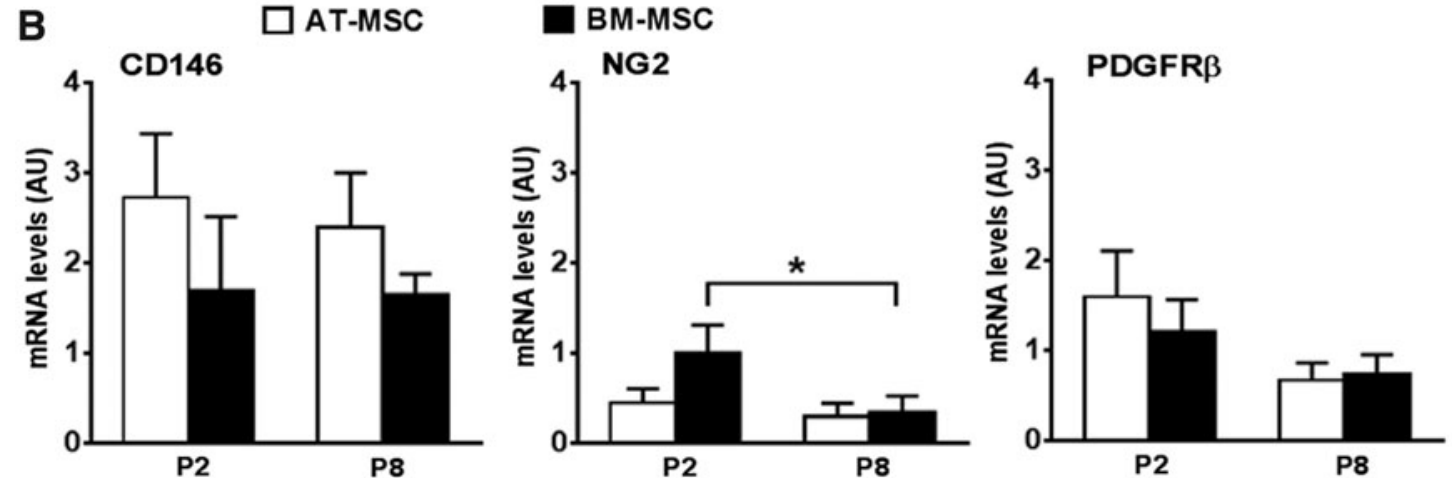

FIG. 4. (A) Flow cytometry histograms showing the proportions of AT-MSCs and BM-MSCs (upper and lower panels, respectively) that are positive for staining with the antibodies CD146-AF647, NG2-APC, and $\alpha$ SMA (with secondary AF405 conjugated). Isotype controls are shown by the gray peak and unstained curves are omitted for simplicity. (B) Results of qPCR analysis of CD146, NG2, and PDGFR $\beta$ in AT-MSCs and BM-MSCs at passages 2 (P2) and 8 (P8). All results are shown as mean \pm SEM; $n=3$ animals. $* P<0.05$.
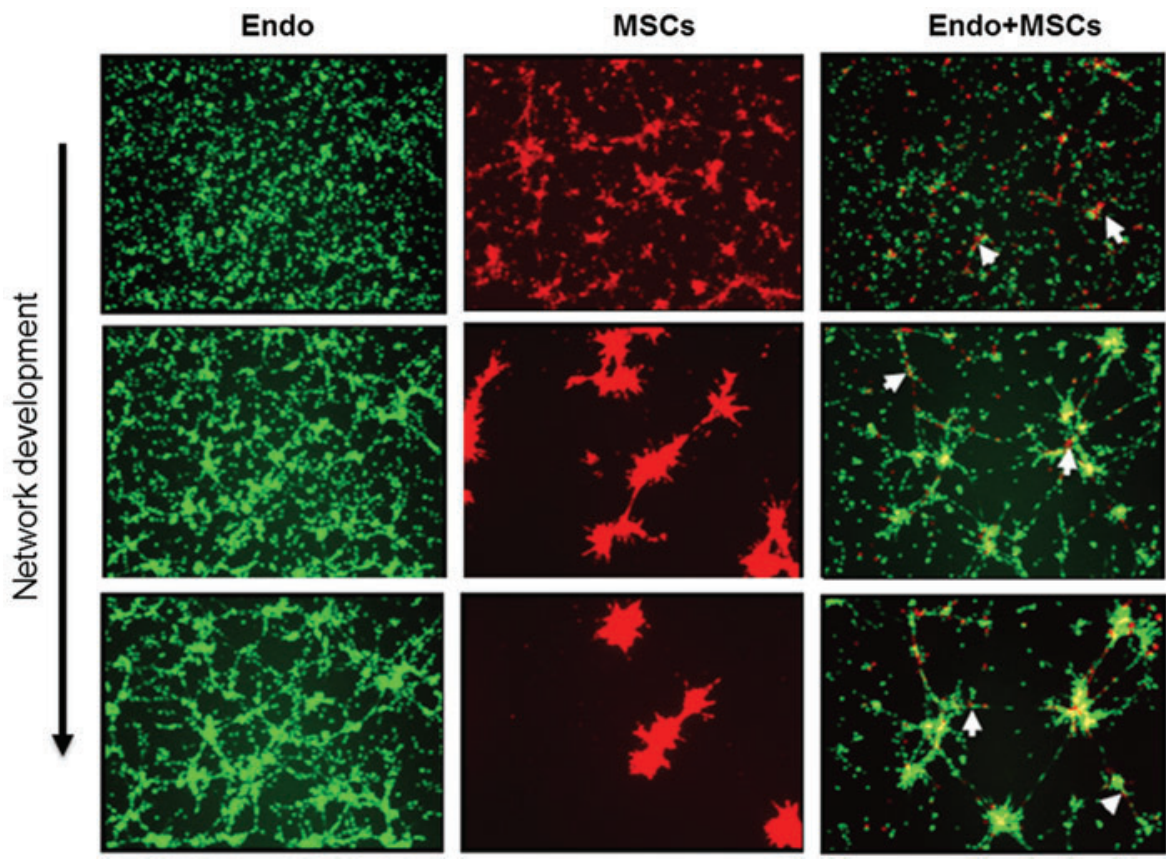

FIG. 5. Microphotographs of cultured equine endothelial cells (Endo, labeled with the green fluorescent PKH67), AT-MSCs (MSCs, labeled with the red fluorescent $\mathrm{PKH} 26$ ) or both cell types together (Endo+MSCs) obtained after seeding on matrigel. Arrows point to MSCs associated with endothelial cells. Color images available online at www .liebertpub.com/scd 
cultured in the absence of endothelia cells organized quickly, forming dense cores of cells that were interlinked by thin bridging extensions, which became more condensed. In cocultures, AT-MSCs tended to colocate with endothelial cells beginning even before a network formed and, importantly, at later stages, AT-MSCs remained closely associated with the endothelial networks, resembling the perivascular location of pericytes in vivo (Fig. 1).

\section{Discussion}

Clinical MSCs are commonly obtained through culture of crude (nonpurified) extracts, typically from BM or AT. Although equine MSCs are widely used clinically, there is a significant lack of knowledge regarding their in vivo origin, identity, and maintenance of original phenotype in culture. This has hindered efforts to characterize equine MSCs as well as exploit their full therapeutic potential. Studies performed in humans $[25,27,30]$ have shown that pericytes, a cell type surrounding small blood vessels throughout the body, express MSC markers both in situ and during culture after isolation. Moreover, cultured pericytes can differentiate into several mesenchymal derivatives, findings all of which implicate pericytes as native precursors of MSCs. However, whether nonpurified equine MSC preparations used clinically maintain original characteristics of pericytes in culture has not been studied. In this project, we investigated the relationship between equine MSCs and pericytes by performing analyses both in equine tissues and in cultured MSCs. Our results shed new light on the nature of these elusive cells by indicating that native pericytes are progenitors of equine MSC populations derived in culture. This conclusion is based on our findings that (1) typical markers of pericytes and MSCs colocalized around small blood vessels in equine AT, (2) cultured equine AT-MSCs and BM-MSCs both expressed pericyte markers over extended culture (up to passage 8), and (3) equine MSCs closely associated with endothelia cell networks in cocultures.

Flow cytometry analyses revealed most cells in both ATMSCs and BM-MSCs preparations contained CD146 and NG2. The presence of CD146 mRNA in equine MSCs has been previously reported [20]. Our results showed a broad distribution in fluorescence intensity of both CD146 and NG2 in equine MSCs, with a small fraction of cells having no or very low expression, indicating cell heterogeneity and/or potential loss of pericyte immunophenotype in culture. Nonetheless, mRNA levels for these markers were sustained up to passage 8, except for NG2 in BM-MSC populations. Around $10 \%$ of MSCs were negative for $\alpha \mathrm{SMA}$, a finding that may be explained by the observation that a subset of pericytes do not naturally express this marker [25,31]. Consistent with their pericyte immunophenotype was the tendency of equine AT-MSCs to closely associate with endothelial cell networks in cocultures, a property that has been reported before for MSCs [32,33] and pericytes [34,35] in human but not in horse. Based on our results showing maintenance of a pericyte phenotype by MSCs over extended culture, it may be desirable to include pericyte markers, for example, CD146, in the panel of markers currently used to immunophenotype equine MSCs. Since perivascular cells expressing CD146 are present in most body tissues [25,30,34], CD146 may serve as a universal MSC marker. In addition, CD146 has been identified as a marker of multipotency in human MSCs [36], which could provide obvious advantages for tissue regeneration purposes.
We detected CD29, CD44, CD90, and CD105 in a majority of cells in AT-MSC and BM-MSC populations, in agreement with many $[18,19,20,37]$ but not all [38] previous studies. A limited number of studies have compared the expression of MSC markers between AT-MSCs and BM-MSCs. In accordance with the present data, no significant differences were found in transcript levels of CD73, CD90, and CD105 [39] between these two cell types, whereas higher expression of CD44 and CD90 was reported for BM-MSCs than for ATMSCs [20]. These discrepancies may be related to the use of different extraction methods, cell culture conditions, analytical techniques, and cell immunophenotyping reagents. As already suggested [19,21], better standardization of techniques used to collect, culture, and analyze equine MSCs is needed before results from different studies can be meaningfully compared.

According to ISCT guidelines, all MSCs should be negative for CD45, and AT-MSCs should additionally lack CD31. Several previous studies did not detect CD45 in equine MSCs using flow cytometry [19,39-41]; however, we (this study) and Radcliffe et al. [18] did detect this marker by qPCR. CD31 (or other endothelial markers) has not been commonly considered in the characterization of equine MSC preparations, in part, because ISCT recommendations on CD31 were just introduced in 2013 [15,16]. Although we did not detect CD31 in our preparations, CD144 (another endothelial marker) was present at low levels in all MSCs. Overall, these results demonstrate the heterogeneity of equine MSCs from different sources and highlight the necessity to use different approaches to fully characterize these preparations, an important consideration because cell heterogeneity may significantly impact the outcome of treatments [36,42].

In conclusion, our results from the analysis of equine tissues and MSC preparations in culture indicate that equine MSCs originate from pericytes and that they maintain a pericyte immunophenotype in culture. Taken together with data available in other species, our results indicate that the isolation and selective culture of pericytes from crude extracts could provide a means to increase the quality and clinical efficacy of equine MSC preparations. Although selective harvesting and in vitro expansion of equine pericytes may be challenging, the findings obtained in this study represent an initial step toward that goal.

\section{Acknowledgments}

We would like to thank Drs. John Keen and Ruth Morgan and the staff at the Pathology Services in the Royal (Dick) School of Veterinary Studies. This work was supported by the Horserace Betting Levy Board (Prj768) and an Institute Strategic Programme Grant from The Biotechnology and Biological Sciences Research Council.

\section{Author Disclosure Statement}

B.P. is coinventor of human perivascular stem cell-related patents filed from University of California, Los Angeles. The other authors declare no competing interests.

\section{References}

1. Smith RKW, ER Garvican and LA Fortier. (2014). The current 'state of play' of regenerative medicine in horses: what the horse can tell the human. Regen Med 9:673-685. 
2. Donadeu FX and CL Esteves. (2016). Stem cells and equine health. www.eurostemcell.org/factsheet/stem-cells-and-equinehealth

3. Pacini S, S Spinabella, L Trombi, R Fazzi, S Galimberti, F Dini, F Carlucci and M Petrini. (2007). Suspension of bone marrow-derived undifferentiated mesenchymal stromal cells for repair of superficial digital flexor tendon in race horses. Tissue Eng 13:2949-2955.

4. Smith R. (2008). Mesenchymal stem cell therapy for equine tendinopathy. Disabil Rehabil 30:1752-1758.

5. Gittel C, J Burk, I Ribitsch and W Brehm. (2011). Efficiency of adipogenic differentiation methods in mesenchymal stromal cells from diverse sources. Regen Med 6:203.

6. Godwin E, N Young, J Dudhia, I Beamish and R Smith. (2012). Implantation of bone marrow-derived mesenchymal stem cells demonstrates improved outcome in horses with overstrain injury of the superficial digital flexor tendon. Equine Vet J 44:25-32.

7. Smith R, M Korda, G Blunn and A Goodship. (2003). Isolation and implantation of autologous equine mesenchymal stem cells from bone marrow into the superficial digital flexor tendon as a potential novel treatment. Equine Vet J 35:99-102.

8. Richardson L, J Dudhia, P Clegg and R Smith. (2007). Stem cells in veterinary medicine-attempts at regenerating equine tendon after injury. Trends Biotechnol 25:409-416.

9. Guest DJ, MR Smith and WR Allen. (2008). Monitoring the fate of autologous and allogeneic mesenchymal progenitor cells injected into the superficial digital flexor tendon of horses: preliminary study. Equine Vet J 40:178-181.

10. Crovace A, L Lacitignola, G Rossi and E Francioso. (2010). Histological and immunohistochemical evaluation of autologous cultured bone marrow mesenchymal stem cells and bone marrow mononucleated cells in collagenase-induced tendinitis of equine superficial digital flexor tendon. Vet Med Int 2010:250978.

11. Schnabel LV, LA Fortier, CW McIlwraith and KM Nobert. (2013). Therapeutic use of stem cells in horses: which type, how, and when? Vet J 197:570-577.

12. Frisbie D and R Smith. (2010). Clinical update on the use of mesenchymal stem cells in equine orthopaedics. Equine Vet J 42:86-89.

13. Clegg PD and GL Pinchbeck. (2011). Evidence-based medicine and stem cell therapy: how do we know such technologies are safe and efficacious? Vet Clin North Am Equine Pract 27:373-382.

14. Taylor SE and PD Clegg. (2011). Collection and propagation methods for mesenchymal stromal cells. Veterinary clinics of North America: Equine Practice 27:263-274.

15. Dominici M, B Le, I Mueller, I Slaper-Cortenbach, F Marini, D Krause, R Deans, A Keating, D Prockop and E Horwitz. (2006). Minimal criteria for defining multipotent mesenchymal stromal cells. The International Society for Cellular Therapy position statement. Cytotherapy 8:315-317.

16. Bourin P, BA Bunnell, L Casteilla, M Dominici, AJ Katz, KL March, H Redl, JP Rubin, K Yoshimura and JM Gimble. (2013). Stromal cells from the adipose tissue-derived stromal vascular fraction and culture expanded adipose tissue-derived stromal/stem cells: a joint statement of the International Federation for Adipose Therapeutics and Science (IFATS) and the International Society for Cellular Therapy (ISCT). Cytotherapy 15:641-648.

17. De Schauwer C, E Meyer, GR Van de Walle and A Van Soom. (2011). Markers of stemness in equine mesenchymal stem cells: a plea for uniformity. Theriogenology 75:14311443.

18. Radcliffe CH, MJ Flaminio and LA Fortier. (2010). Temporal analysis of equine bone marrow aspirate during establishment of putative mesenchymal progenitor cell populations. Stem Cells Dev 19:269-282.

19. De Schauwer C, S Piepers, GR Van de Walle, K Demeyere, MK Hoogewijs, JL Govaere, K Braeckmans, A Van Soom and E Meyer. (2012). In search for cross-reactivity to immunophenotype equine mesenchymal stromal cells by multicolor flow cytometry. Cytometry A 81:312-323.

20. Ranera B, J Lyahyai, A Romero, FJ Vázquez, AR Remacha, ML Bernal, P Zaragoza, C Rodellar and I MartínBurriel. (2011). Immunophenotype and gene expression profiles of cell surface markers of mesenchymal stem cells derived from equine bone marrow and adipose tissue. Vet Immunol Immunopathol 144:147-154.

21. Burk J, SF Badylak, J Kelly and W Brehm. (2013). Equine cellular therapy-from stall to bench to bedside? Cytometry Part A 83A:103-113.

22. Armulik A, G Genove and C Betsholtz. (2011). Pericytes: developmental, physiological, and pathological perspectives, problems, and promises. Dev Cell 21:193-215.

23. Chen WC, TS Park, IR Murray, L Zimmerlin, L Lazzari, J Huard and B Peault. (2013). Cellular kinetics of perivascular MSC precursors. Stem Cells Int 2013:983059.

24. Murray IR, CC West, WR Hardy, AW James, TS Park, A Nguyen, T Tawonsawatruk, L Lazzari, C Soo and B Peault. (2014). Natural history of mesenchymal stem cells, from vessel walls to culture vessels. Cell Mol Life Sci 71:1353-1374.

25. Crisan M, S Yap, L Casteilla, C-W Chen, M Corselli, TS Park, G Andriolo, B Sun, B Zheng, et al. (2008). A perivascular origin for mesenchymal stem cells in multiple human organs. Cell Stem Cell 3:301-313.

26. Esteves CL, V Kelly, V Bégay, SG Lillico, A Leutz, JR Seckl and KE Chapman. (2013). Stable conditional expression and effect of C/EBP $\beta$-LIP in adipocytes using the pSLIK system. J Mol Endocrinol 51:91-98.

27. Crisan M, C-W Chen, M Corselli, G Andriolo, L Lazzari and B Péault. (2009). Perivascular multipotent progenitor cells in human organs. Ann N Y Acad Sci 1176:118-123.

28. Sobiesiak M, K Sivasubramaniyan, C Hermann, C Tan, M Orgel, S Treml, F Cerabona, P de Zwart, U Ochs, et al. (2010). The mesenchymal stem cell antigen MSCA-1 is identical to tissue non-specific alkaline phosphatase. Stem Cells Dev 19:669-677.

29. Zimmerlin L, VS Donnenberg, JP Rubin and AD Donnenberg. (2013). Mesenchymal markers on human adipose stem/progenitor cells. Cytometry A 83:134-140.

30. Zimmerlin L, VS Donnenberg, ME Pfeifer, EM Meyer, B Peault, JP Rubin and AD Donnenberg. (2010). Stromal vascular progenitors in adult human adipose tissue. Cytometry A 77:22-30.

31. Nehls V and D Drenckhahn. (1993). The versatility of microvascular pericytes: from mesenchyme to smooth muscle? Histochemistry 99:1-12.

32. Li Q and Z Wang. (2013). Influence of mesenchymal stem cells with endothelial progenitor cells in co-culture on osteogenesis and angiogenesis: an in vitro study. Arch Med Res 44:504-513.

33. Aguirre A, JA Planell and E Engel. (2010). Dynamics of bone marrow-derived endothelial progenitor cell/mesenchymal stem cell interaction in co-culture and its implications in angiogenesis. Biochem Biophys Res Commun 400:284-291. 
34. Chen WC, JE Baily, M Corselli, M Diaz, B Sun, G Xiang, GA Gray, J Huard and B Peault. (2015). Human myocardial pericytes: multipotent mesodermal precursors exhibiting cardiac specificity. Stem Cells 33:557-573.

35. Blocki A, Y Wang, M Koch, P Peh, S Beyer and P Law. (2013). Not all MSCs can act as pericytes: functional in vitro assays to distinguish pericytes from other mesenchymal stem cells in angiogenesis. Stem Cells Dev 22:2347-2355.

36. Russell KC, DG Phinney, MR Lacey, BL Barrilleaux, KE Meyertholen and KC O'Connor. (2010). In vitro high-capacity assay to quantify the clonal heterogeneity in trilineage potential of mesenchymal stem cells reveals a complex hierarchy of lineage commitment. Stem Cells 28:788-798.

37. Barberini D, N Freitas, M Magnoni, L Maia, A Listoni, M Heckler, M Sudano, M Golim, F Landim-Alvarenga and R Amorim. (2014). Equine mesenchymal stem cells from bone marrow, adipose tissue and umbilical cord: immunophenotypic characterization and differentiation potential. Stem Cell Res Ther 5:25.

38. Paebst F, D Piehler, W Brehm, S Heller, C Schroeck, A Tarnok and J Burk. (2014). Comparative immunophenotyping of equine multipotent mesenchymal stromal cells: An approach toward a standardized definition. Cytometry A 85:678-687.

39. Ranera B, L Ordovas, J Lyahyai, M Bernal, F Fernandes, A Remacha, A Romero, F Vazquez, R Osta and C Cons. (2012). Comparative study of equine bone marrow and adipose tissue-derived mesenchymal stromal cells. Equine Vet J 44:33-42.

40. Braun J, A Kurtz, N Barutcu, J Bodo, A Thiel and J Dong. (2013). Concerted regulation of CD34 and CD105 accompanies mesenchymal stromal cell derivation from human adventitial stromal cell. Stem Cells Dev 22:815-827.

41. Choudhary RK. (2014). Mammary stem cells: expansion and animal productivity. J Anim Sci Biotechnol 5:36.

42. Kassem M and P Bianco. (2015). Skeletal Stem Cells in Space and Time. Cell 160:17-19.

Address correspondence to: Dr. Cristina L. Esteves

The Roslin Institute University of Edinburgh Easter Bush Midlothian

EH25 9RG Edinburgh United Kingdom

E-mail: cristina.esteves@ roslin.ed.ac.uk

Received for publication January 20, 2017

Accepted after revision April 4, 2017

Prepublished on Liebert Instant Online April 4, 2017 